\title{
Discrimination of complex visual stimuli in Cebus apella: identity matching with pictures
}

\author{
Olavo F. Galvão, Paulo S. D. Soares Filho, Hernando B. Neves Filho and Milena M. Nagahama \\ Universidade Federal do Pará, Brazil
}

\begin{abstract}
Do capuchin monkeys respond to photos as icons? Do they discriminate photos of capuchin monkeys' faces? Looking for answers to these questions we trained three capuchin monkeys in simple and conditional discrimination tasks and tested the discriminations when comparison stimuli were partially covered. Three capuchin monkeys experienced in simultaneous simple discrimination and IDMTS were trained with repeated shifts of simple discriminations (RSSD), with four simultaneous choices, and IDMTS ( $1 \mathrm{~s}$ delay, 4 choices) with pictures of known capuchins monkeys' faces. All monkeys did discriminate the pictures in both procedures. Performances in probes with partial masks with one fourth of the stimulus hidden were consistent with baseline level. Errors occurred when a picture similar to the correct one was available among the comparison stimuli, when the covered part was the most distinct, or when pictures displayed the same monkey. Capuchin monkeys do match pictures of capuchin monkeys' faces to the sample. The monkeys treated different pictures of the same monkey as equivalent, suggesting that they respond to the pictures as icons, although this was not true to pictures of other monkeys. Subsequent studies may bring more evidence that capuchin monkeys treat pictures as depictions of real scenes. Keywords: simple discrimination, conditional discrimination, matching to sample, iconic representation, photos as stimuli, Cebus apella.
\end{abstract}

Received 13 February 2009; received in revised form 23 March 2009; accepted 8 April 2009. Available online 29 June 2009

\section{Introduction}

Researchers de Waal and Pokorny (2008) reported that male chimpanzees respond differently to pictures of familiar and unfamiliar chimpanzees. The belief that chimpanzees respond to the pictures as icons is implicit. But do they? The problem of knowing the function of images for monkeys was first studied by Gallup (1970) measuring the reaction of apes to their own mirror image with a white mark on the forehead. Later, InoueNakamura (1997) showed that Cebus apella present higher frequency of mirror observation and higher proportion of social behaviors directed to the mirror, compared to other primates. Anderson, Kuroshima, Paukner and Fujita (2008) reported that capuchin monkeys react to real-time movies of themselves, although without explicit acknowledgement that they recognize themselves, they prefer real-time to 1 s-delayed movies. While apes were the only primates

Olavo F. Galvão, Paulo S. D. Soares Filho, Hernando B. Neves Filho and Milena M. Nagahama, Theory and Research of Behavior Graduate Program, Federal University of Pará State. Correspondence regarding this article should be directed to: Olavo F. Galvão, Trav. Castelo Branco, 1116, Apt. 1004, Belém, PA, Brazil, CEP 66063-000. E-mail: olavo@ pq.cnpq.br. Phone 559132018475. that passed in Gallup's test of self recognition, the social behavior of capuchin monkeys in front of mirrors and movie-screens is evidence that the observer's mirror image is equivalent to the presence of an individual (Paukner, Anderson, \& Fujita, 2004).

Pascalis and Bachevalier (1998) with humans and monkeys (Macaca mulata), and Dufour, Pascalis and Petit (2006) with humans, Tonkean macaques and brown capuchins, showed that the observation of a new face was predominant (longer) during the exhibition of a pair of faces (one previously shown) of the same species, and that this effect was restricted to faces of the same species. A remarkable aspect of these studies was the use of black and white pictures as stimuli. Another remarkable face recognition effect is the independent measurement of responses of temporal neurons that respond specifically to facial pictures and sketches (Sagiv \& Bentin, 2001).

"Normal" humans respond to complex visual stimuli scanning the whole stimulus, and if part of the stimulus is hidden, they respond to the remaining as to the complete figure (Dube et al., 2003). In the context of delayed IDMTS, Dickson, Wang, Lombard and Dube (2006) demonstrated that individuals with learning disabilities may restrict the identity relation to parts of a complex stimulus, choosing the incorrect comparison when that part is added to the incorrect 
choice, for example. This performance is called over selectivity or restricted control.

Capuchin monkeys can show successful performances in simple and conditional discrimination studies with arbitrary visual stimuli (Barros, Galvão, Brino, Goulart, \& McIlvane, 2005; Barros, Galvão, \& McIlvane, 2002; Goulart, Mendonça, Barros, Galvão, $\&$ Mcllvane, 2005), and photographs were used successfully with capuchins in simple discrimination studies (Beran et al., 2008; D'amato \& Van Sant, 1988). Demonstration of recognition of photos of faces in an identity matching to sample format could be taken as evidence that they can respond to pictures as icons that are related to the conspecific individual photographed.

Similarly to the recognition studies, this paper intends to investigate some variables involved in using pictures as stimuli in identity conditional discrimination with capuchin monkeys. The use of pictures of conspecifics' faces in simple discrimination, repeated shifts of simple discrimination, and conditional discrimination (IDMTS) with capuchins with a history of generalized matching to sample with arbitrary black-gray-white drawings as stimuli is a preparation for the demonstration of equivalence between photographs and the items depicted in an object-picture matching procedure.

This study also addresses the possibility that capuchin monkeys may match by identity small parts of the nominal stimulus (over selectivity). Trying to verify this, after the IDMTS baseline was trained, the stimuli were partially covered to verify eventual restricted control.

\section{Method}

\section{Subjects}

The participants were three male adult capuchin monkeys (Cebus apella): M07, ET; M12, Cotoh; M15, Louis Dubois, living in the same facility but in separate cages. The monkeys had previous experience in simple discrimination and matching to sample with black-gray-white drawings.

\section{Apparatus}

The experimental sessions of subjects M07 and M15 were performed in a different experimental chamber and using a different software (EAM v. 4.0) from those used in the sessions of subject M12 (VAICOM). Each chamber was equipped with a touch sensitive monitor. A $190 \mathrm{mg}$ pellet dispenser (MED Associates Inc ENV203-190) provided one pellet per correct response. The software is available from the authors by request.

\section{Stimuli}

All the stimuli were in *.bmp format, with 24 bits of color and $93 \times 93$ pixels resolution. All of them were pictures of faces of conspecific monkeys (taken from monkeys that lived in the same animal house). Figure 1 shows the pictures used as stimuli grouped in four sets, corresponding to four baselines (A, B, C and D) of four stimuli trained separately. The alphanumeric code and the name of the monkey depicted are shown above each picture. There were different pictures of the same monkey. No pictures of the experimental subjects were used. The $5 \times 5 \mathrm{~cm}$ stimuli were presented on a white background in any of nine locations in a $3 \times 3$ (three columns by three lines) matrix in the touch sensitive screen.

\section{General Procedure}

The sessions consisted of discrete trials separated by an inter-trial interval (ITI). Touching the correct stimulus (S+) twice was followed by delivery of a $190 \mathrm{mg}$ pellet of the preferred flavor for each subject, and an ITI of six seconds followed. Responses (FR1) directed to any one of the incorrect choices (S-) produced only the ITI and the same trial was repeated (correction procedure). In the simple discrimination procedure two or four stimuli were presented simultaneously in each trial, in varying locations; sessions ended when the maximum number of trials was reached or when six consecutive correct trials occurred. When the criterion level was reached the correct stimulus was changed. In reversals of simple discrimination the $\mathrm{S}+$ was changed in shifts. In the IDMTS procedure, a stimulus was presented in any of the nine positions in the monitor. Two touches on the sample were followed by the disappearance of the sample and the immediate presentation of the comparison stimuli, in varying locations. The number of choices in a given trial varied from two to four. After the learning criterion was reached, the partial mask procedure was introduced. One-fourth of both comparison stimuli was covered by a blank square.

The mask covered each of the four stimulus quadrants, A, B, C and D (Figure 2). In a given trial, all comparison stimuli had the same quadrant covered.

The procedure was divided in six phases. Participants M07 and M15 were exposed to all phases except for phases 6 and 8; participant M12 begun at phase 6 (see Table 1).

Phase 1 (reversal of visual simultaneous simple discrimination with two choices): Set A was divided in two two-stimuli sets (A1-A2 and A3-A4) for simple discrimination training. Each stimulus was the $\mathrm{S}+$ in turns. When a stimulus was the $\mathrm{S}+$, the other stimulus was the S-. Sessions ended with 36 trials or six consecutive correct responses, whichever came first. Phase 1 ended after two sessions with each S+ (A1, A2, A3, A4).

Phase 2: Same as Phase 1, except for a blank square substituting the $\mathrm{S}+$ in one third of the trials and the $\mathrm{S}$ - in the other third of the trials (blank comparison probes).

Phase 3: Same as Phase 1.

Phase 4: Repeated shifts of simple discrimination (RSSD) with four choices using stimuli set A. In this phase, when a stimulus was the $\mathrm{S}+$, the other three were S-.

Phase 5: Same as previous one plus Partial Mask - a blank square covering one-fourth of the stimuli, in one 


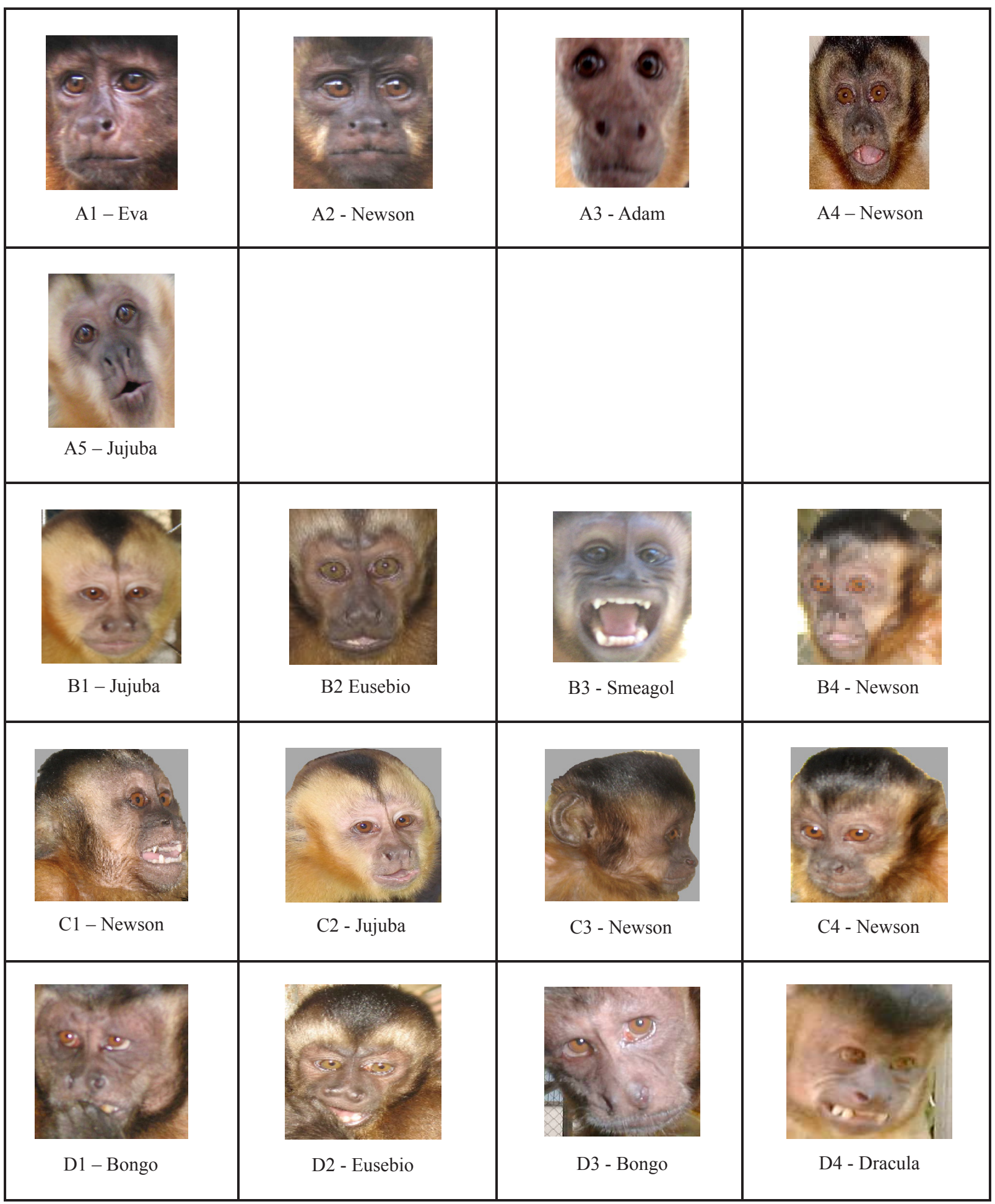

Figure 1. Stimuli used in the experiment, divided in four sets (A, B, C, D) of four stimuli. Conditional discrimination tasks involved each stimulus of a set in turns as sample; comparisons were stimuli of the same set. A5 eventually substituted A1.

trial the four stimuli had the same quadrant covered; in subsequent trials every quadrant was covered in turns.

Phase 6: Special procedure for participant M12 only. Consisting basically of repeated shifts of simple discrimination, but in a given session the $\mathrm{S}+$ of the previous session was not used as S-; trials had three choices until all the stimuli were $\mathrm{S}+$ twice. Sessions in this phase had 36 trials, and the criterion to move on to the next phase was six consecutive correct responses in each session.

Phase 7: IDMTS, four choices, zero delay, with stimuli sets A, B, C, D, successively. After training 


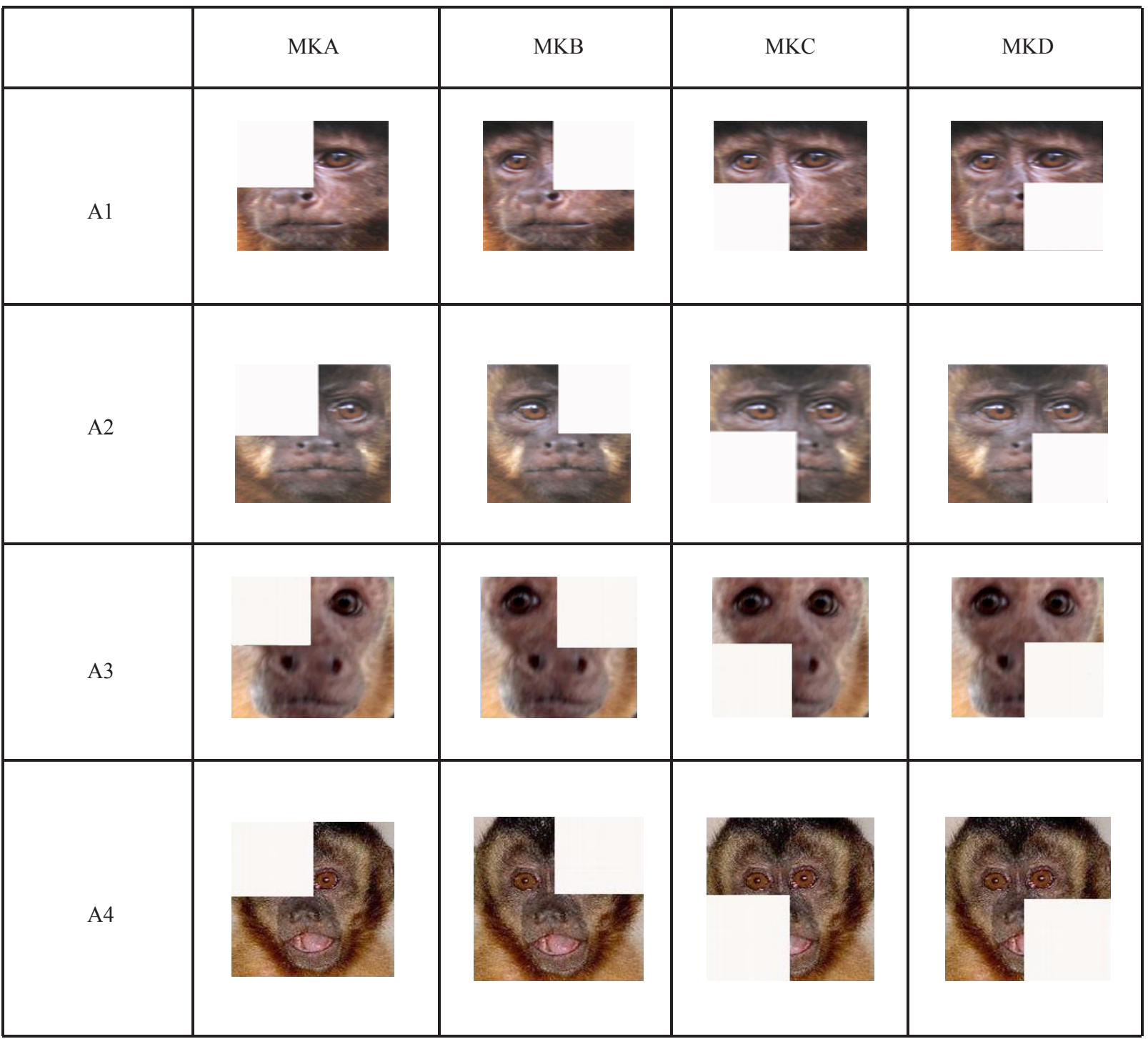

Figure 2. Stimulus set A with Partial Mask (General Procedure).

with set A, the first session with each new stimulus set had 24 baseline and 12 probe trials with stimuli from the next training set before training with the new set (insertion probe session). Sessions had 36 trials and the criterion to move on to the next phase was more than $90 \%$ correct responses, and less than two errors per trial type. Correct responses in the first trial, in each insertion probe trial, and more than $75 \%$ of correct responses in all the 12 probe trials were regarded as performances of generalized IDMTS.

Phase 8: Participant M12 only. IDMTS, zero delay, four choices with partial mask, successively with stimulus sets A, B, C, D. After reaching criterion with each stimulus set, a session with 12 trials with a partial mask and 24 baseline trials was performed before the insertion probe session with the next set.

Phase 9: IDMTS, zero delay, four choices. Sessions with trials of sets A, B, C and D, shuffled. In a given trial, comparisons were the four stimuli from the same set as the sample. Sessions had 48 trials; criteria to end the phase were at least $90 \%$ correct responses and less than two errors per trial type.

Phase 10: IDMTS sessions with trials of sets A, B, C and D, shuffled as in Phase 9, with partial mask probes in comparisons (32 trials with a partial mask, 16 without), as in Phase 5 in a given trial the partial mask was in the same quadrant on each comparison stimulus. Each partial mask position was presented four times. Probes were completed after eight 48 -trial sessions, with 256 partial mask and 128 baseline trials.

\section{Results}

In Phase 1 (two-choice simple discrimination reversals) participants M07 and M15 reached the criterion of ending two consecutive sessions with six consecutive correct trials for each $\mathrm{S}+$ in 14 and 17 sessions, respectively.

In Phase 2 (probe trials with blank comparison substituting the $\mathrm{S}+$ or the $\mathrm{S}$-) inconsistent results were 
Table 1. Sequence of phases and participants. RSSD = Repeated shifts of simple discrimination; IDMTS = Identity Matching to Sample.

\section{Phase}

1 Reversals of simple discrimination, Set A

2 Simple discrimination, blank comparison probes

3 Re-training of simple discrimination, Set A

4 RSSD, four choices

5 RSSD, four choices, partial mask

6 RSSD, three and four choices

7 IDMTS, Sets A, B, C, D

8 Partial mask probes in IDMTS training of each set

9 Sets A, B, C, D mixed in the same session

0 Partial mask probes

\section{Participants}

M07 M15 M12

found. Baseline was maintained, but in 12 probe trials M07 never chose the mask, and M15 performance was close to chance performance ( 7 of 12 correct).

In Phase 3 (retraining of reversals of simple discrimination) M07 and M15 reached the criterion of ending the session with six consecutive correct responses in four consecutive reversals within four and 11 sessions, respectively.

In Phase 4 (four choices), M07 and M15 took five and seven sessions respectively to reach the criterion of four sessions ending with six consecutive correct choices.

In Phase 5 (four choices, partial mask), M07 and M15 took 22 and 23 sessions respectively to reach the criterion. Although reaching it, M15 was choosing A2 when $\mathrm{A} 1$ was $\mathrm{S}+$ and vice versa when a partial mask was in quadrant C (Figure 3).

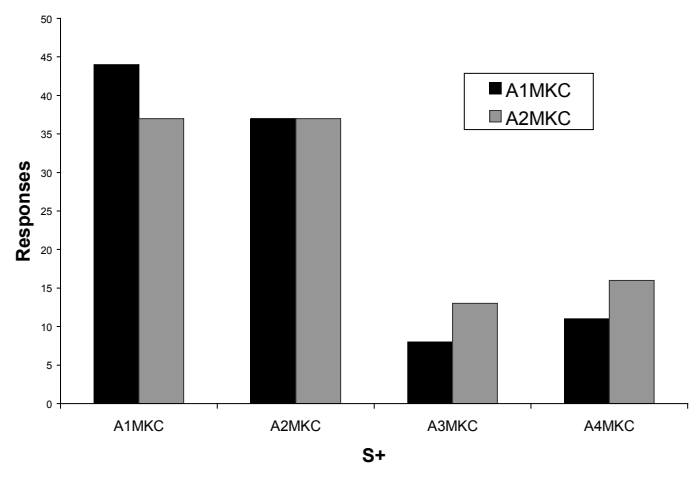

Figure 3. Number of responses in $\mathrm{A} 1 / \mathrm{MKC}$ and $\mathrm{A} 2 / \mathrm{MKC}$ in trials with each $\mathrm{S}+$ for participant M15 in reversals of simple discrimination with partial mask (Phase 5).
Phase 6: Exposed to repeated shifts of simple discrimination, participant M12 reached the criterion of six consecutive correct trials in all sessions, and took eight sessions to reach the criterion to end this phase.

In Phase 7 (IDMTS with sets A, B, C and D), training with set A, participants M07, M12 and M15 met the criterion of $90 \%$ correct responses respectively in 25,11 , and 13 sessions. Similar to the results in Phase 6 , subjects M07 and M15 systematically chose A1 when A2 was the sample, and vice versa (Table 2). Because of these results, A1 was replaced by $\mathrm{A} 5$ for all participants (Figure 1). With stimulus set B, M07, M12, and M15 took seven, 25, and six sessions to meet the criterion. With stimuli set C, M07, M12, and M15 took 13, 10, and 13 sessions to reach the criterion; and to stimulus set D, M07, M12, and M15 took 10, 10, and seven sessions to

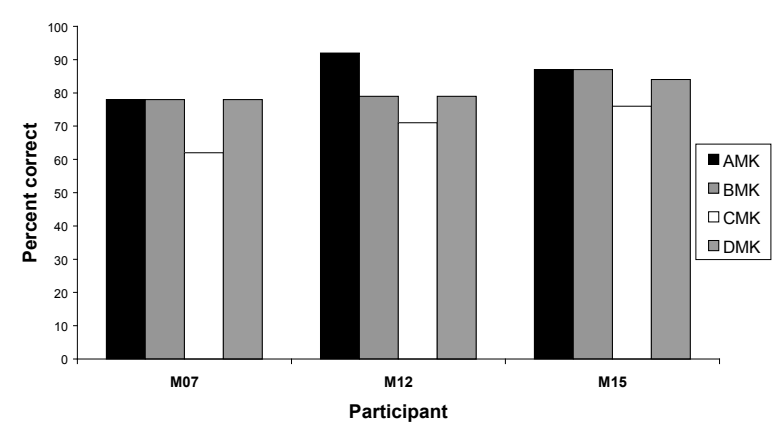

Figure 4. Percent correct responses in all trials in IDMTS with partial mask (Phase 10). 
Table 2. Number of responses to each comparison, by sample, in the first four sessions of IDMTS with stimulus set A.

\begin{tabular}{|c|c|c|c|c|c|c|c|c|}
\hline \multirow{3}{*}{ Sample } & \multicolumn{8}{|c|}{ Comparison } \\
\hline & $\mathrm{A} 1$ & A2 & $\mathrm{A} 3$ & A4 & A1 & A2 & A3 & A4 \\
\hline & \multicolumn{4}{|c|}{ M07 } & \multicolumn{4}{|c|}{ M15 } \\
\hline A1 & 39 & 31 & 22 & 15 & 20 & 42 & 11 & 14 \\
\hline $\mathrm{A} 2$ & $\underline{39}$ & 40 & 22 & 23 & $\underline{31}$ & 21 & 7 & 6 \\
\hline A3 & 16 & 30 & 60 & 14 & 2 & 3 & 24 & 14 \\
\hline A4 & 30 & 35 & 27 & 49 & 1 & 2 & 5 & 29 \\
\hline Total & 124 & 136 & 131 & 101 & 54 & 68 & 47 & 63 \\
\hline
\end{tabular}

reach the criterion. In the first session with 12 trials of the new set (insertion probe session), only M15 showed generalized IDMTS results with stimulus set D.

During IDMTS with set D systematic errors occurred when D1 and D3 were samples. M15 and M07 touched D3 when D1 was sample, and vice versa (see Table 3). These two stimuli were different pictures of the same monkey

In Phase 8 (IDMTS, stimulus set with partial mask), the performance of M12 in the trials with a partial mask was at $94 \%$ correct responses for set A, $68 \%$ for set B, $50 \%$ for set $\mathrm{C}$, and $43 \%$ for set $\mathrm{D}$.

In Phase 9 (stimulus sets A, B, C, D mixed in the same session), M07, M12, M15 reached the criterion in 15 , three, and five sessions. After learning the conditional discrimination within each picture set, they maintained correct performances when trials of the four sets were interspersed in the same session.

In Phase 10 (IDMTS with stimulus sets A, B, C, $\mathrm{D}$ mixed in the same session with partial masks), the number of sessions planned was eight. Performance was above $90 \%$ correct in the four last sessions for all the three participants. In probe trials the overall performance was approximately $80 \%$ (See Figure 4).
Table 4 shows selected results of the four trials with partial mask for trial types with more than $50 \%$ of errors in the same stimulus. The " $\mathrm{X}$ " stands for incorrect choices and the "C" for correct choices, between parentheses are the stimuli chosen in incorrect trials.

Even with consistent errors in partial mask trials, there was no significant loss of performance in any of the participants.

\section{Discussion}

Because all three participants reached performance criteria in repeated shifts of simultaneous simple discrimination and identity, zero-delay, conditional discrimination (IDMTS) with sets of four pictures of capuchins monkeys' faces, we can say that complex stimuli as photographs can be used in discrimination problems with capuchin monkeys. Further studies are necessary to determine the relation between stimulus control by pictures and the objects they represent.

In the probe trials with blank comparison substituting the S+ or the S- (Phase 2) it was not possible to infer about

Table 3. Responses in each comparison per sample, during the sessions of IDMTS with stimulus set D. In each cell the sequence of numbers correspond to responses of participants M07, M12, M15, in this order.

\begin{tabular}{|c|c|c|c|c|}
\hline \multirow{2}{*}{ Sample } & \multicolumn{4}{|c|}{ Comparison } \\
\hline & D1 & D2 & D3 & D4 \\
\hline D1 & $54,56,52$ & $14,3,6$ & $38,14,3$ & $6,0,5$ \\
\hline D2 & $6,0,3$ & $80,59,60$ & $6,2,0$ & $3,12,0$ \\
\hline D3 & $39,11,10$ & $19,4,2$ & $50,55,56$ & $11,3,1$ \\
\hline D4 & $4,4,0$ & $6,5,1$ & $4,0,4$ & $82,55,62$ \\
\hline
\end{tabular}


Table 4. Performance in selected probe trials with partial mask. Mask Location quadrant A to D. C = Correct; $\mathrm{X}=\mathrm{Incorrect}$; between parenthesis chosen stimulus.

\begin{tabular}{cccccc}
\hline Subject & Sample/Mask Location & First & Second & Third & Fourth \\
\hline M07 & B2/MKA & $\mathrm{X}(\mathrm{B} 1)$ & $\mathrm{X}(\mathrm{B} 4, \mathrm{~B} 4, \mathrm{~B} 1, \mathrm{~B} 4)$ & $\mathrm{X}(\mathrm{B} 1)$ & $\mathrm{C}$ \\
& $\mathrm{C} 1$ MKD & $\mathrm{X}(\mathrm{C} 2, \mathrm{C} 4, \mathrm{C} 3)$ & $\mathrm{X}(\mathrm{C} 3, \mathrm{C} 2, \mathrm{C} 4)$ & $\mathrm{X}(\mathrm{C} 2)$ & $\mathrm{C}$ \\
& $\mathrm{C} 3 / \mathrm{MKD}$ & $\mathrm{X}(\mathrm{C} 4, \mathrm{C} 2)$ & $\mathrm{X}(\mathrm{C} 1, \mathrm{C} 2, \mathrm{C} 4, \mathrm{C} 1$, & $\mathrm{C}$ & $\mathrm{X}(\mathrm{C} 1)$ \\
& C4/MKB & $\mathrm{X}(\mathrm{C} 3, \mathrm{C} 2)$ & $\mathrm{X}(\mathrm{C} 3, \mathrm{C} 2)$ & $\mathrm{C}$ & $\mathrm{X}(\mathrm{C} 1)$ \\
& $\mathrm{D} 1 / \mathrm{MKC}$ & $\mathrm{X}(\mathrm{D} 3, \mathrm{D} 2)$ & $\mathrm{C}$ & $\mathrm{X}(\mathrm{D} 3)$ & $\mathrm{X}(\mathrm{D} 3, \mathrm{D} 4, \mathrm{D} 4, \mathrm{D} 2)$ \\
$\mathrm{M} 12$ & $\mathrm{C} 3 \mathrm{MKC}$ & $\mathrm{C}$ & $\mathrm{X}(\mathrm{C} 4, \mathrm{C} 1)$ & $\mathrm{X}(\mathrm{C} 4)$ & $\mathrm{X}(\mathrm{C} 4, \mathrm{C} 2)$ \\
& $\mathrm{C} 4 \mathrm{MKC}$ & $\mathrm{X}(\mathrm{C} 1)$ & $\mathrm{X}(\mathrm{C} 1, \mathrm{C} 2, \mathrm{C} 3)$ & $\mathrm{X}(\mathrm{C} 1, \mathrm{C} 3)$ \\
$\mathrm{M} 15$ & $\mathrm{~B} 1 / \mathrm{MKA}$ & $\mathrm{X}(\mathrm{B} 4)$ & $\mathrm{C}$ & $\mathrm{X}(\mathrm{B} 4, \mathrm{~B} 4)$ & $\mathrm{X}(\mathrm{B} 4)$ \\
& $\mathrm{C} 3 / \mathrm{MKD}$ & $\mathrm{X}(\mathrm{C} 2)$ & $\mathrm{C}(\mathrm{C} 2)$ & $\mathrm{X}(\mathrm{C} 1)$ \\
& $\mathrm{D} 3 / \mathrm{MKC}$ & $\mathrm{C}$ & $\mathrm{X}(\mathrm{D} 1)$ & $\mathrm{C}$ & $\mathrm{X}(\mathrm{D} 1)$ \\
\hline
\end{tabular}

stimulus control, because incorrect choices occurred in both trial types, with mask on S+ and S-. Similar to the results of experiment 1 in Goulart et al., (2005).

Subject M15 showed generalized IDMTS in Phase 7, the first replication with pictures of Galvão et al. (2005) who reported generalized IDMTS with black and white drawings as stimuli. Concerning the confusion between two different pictures of the same monkey in Phase 7 (Bongo: D1, D3), which could be interpreted as indicative of iconicity, we need further investigation because the discrimination of different pictures of other monkey was unaffected (Newson: C1, C3, C4).

The use of partial masks covering one fourth of the pictures was intended to assess whether monkeys would exhibit restricted control in a picture discrimination task. Restricted control, i.e., responses under the control of part of the stimulus, is a phenomenon demonstrated in autistic individuals (Dickson, Deutsch, Wang, \& Dube, 2006; Dube \& Mcllvane, 1999). The discrimination problems presented by subjects M07 and M15, between A1 and A2, can not be attributed to restricted control, because discrimination between pictures happened when the most conspicuous differences between the stimuli were presented. Discrimination problems occurred when the not covered parts were the most similar. This is a normal discrimination problem already demonstrated elsewhere (Sidman \& Stoddard, 1967), a phenomenon that is different from the over selectivity reported in autism. Decrease in performance in conditional discrimination when a partial mask was in use in Phase 10, again, could not be interpreted as indicative of over selectivity, because general performance was still high and no consistent pattern of errors was found.

The insertion probes of sets B, C and D indicated that with the procedure used there was no immediate transfer of identity matching to the new pictures. Participant
M15 showed a gradual improvement of performance, reaching the criterion for generalized identity with the insertion probe for stimulus set D.

The procedure used in this experiment can be used in the experimental approach to the study of symbolic behavior to describe the conditions that produce this kind of repertoire, and eventually to develop baselines for the analysis of pre-symbolic behavior at biobehavioral and neural levels (Galvão, Soares-Filho, Barros, \& Souza, 2008).

Using sets with larger number of pictures and trials with more simultaneously presented pictures as comparison stimuli is a possible follow-up study to measure the discriminative potential of these stimuli.

\section{Acknowledgements}

Support to this research was provided by $\mathrm{CNPq}$ (Research Productivity Fellowship P. 15245-5/200 and undergraduate grants P. 485135/2006-0; P. 478821 2003-4), FINEP (FNDCT/CT-INFRA: EDITAL 002/03, Conv. FADESP 01.04.0200.00 02/2003), and NIH (Convênio 681 UMW/UFPA/FADESP, 2002).

\section{References}

Anderson, R., Kuroshima, H., Paukner, A., \& Fujita, K. (2008). Capuchin monkeys (Cebus apella) respond to video images of themselves. Animal Cognition, 11, 109-116.

Barros, R.S., Galvão, O.F., Brino, A.L.F., Goulart, P.R.K., \& McIlvane, W.J. (2005). Variáveis de procedimento na pesquisa sobre classes de equivalência: contribuições para o estudo do comportamento simbólico. Revista Brasileira de Análise do Comportamento, 1, 15-27.

Barros, R.S., Galvão, O.F., \& McIlvane, W.J. (2002). Generalized Identity Matching-to-Sample in Cebus apella. The Psychological Record, 52, 441-460.

Beran, M.J., Klein, E.D., Evans, T.A., Chan, B., Flemming, T.M., Harris, E.H., Washburn, D.A., \& Rumbaugh, D.M. (2008) Discrimination reversal learning in capuchin monkeys (Cebus apella). The Psychological Record, 58, 3-14.

D'amato, M.R., \& Van Sant, P. (1988). The person concept in monkeys 
(Cebus apella). Journal of Experimental Psychology, 14, 43-55.

de Waal, F.B.M., \& Pokorny, J.J. (2008). Faces and Behinds: Chimpanzee Sex Perception. Advanced Science Letters, 1, 99-103.

Dickson, C.A., Deutsch, C.K., Wang, S.S., \& Dube, W.V. (2006). Matching-to-sample assessment of stimulus overselectivity in students with intellectual disabilities. American Journal on Mental Retardation, 111, 447-453.

Dickson, C.A., Wang, S.S., Lombard, K.M., \& Dube, W.V. (2006). Overselective stimulus control in residential school students with intellectual disabilities. Research in Developmental Disabilities, 27, 618-631.

Dube, W.V., Lombard, K.M., Farren, K.M., Flusser, D.S., Balsamo, L.M., Fowler, T.R., \& Tomanari, G.Y. (2003). Stimulus overselectivity and observing behavior in individuals with mental retardation. In: Soraci, S., \& Murata-Soraci, K. (eds.)._Visual information processing (pp. 109-123). Westport, CT: Praeger.

Dube, W.V., \& McIlvane, W.J. (1999). Reduction of stimulus overselectivity with nonverbal differential observing responses. Journal of Applied Behavior Analysis, 32, 25-33.

Dufour, V., Pascalis, O., \& Petit, O. (2006). Face processing limitation to own species in primates: a comparative study in brown capuchins,
Tonkean macaques and humans. Behavioural Processes, 73, 107-113. Gallup, G.G. (1970). Chimpanzees: self-recognition. Science, 167, 86-87.

Galvão, O.F., Soares-Filho, P.S.D., Barros, R.S., \& Souza, C.B.A. (2008). Matching to sample as model of symbolic behavior for biobehavioral investigation. Reviews in the Neurosciences, 19, 149-156.

Goulart, P.R.K., Mendonça, M.B., Barros, R.S., Galvão, O.F., \& McIlvane, W.J. (2005). A note on Select and Reject Controlling Relations in the Simple Discrimination of Capuchin Monkeys (Cebus apella). Behavioural Processes 69, 295-302.

Inoue-Nakamura, N. (1997). Mirror self recognition in nonhuman primates: a phylogenetic approach. Japanese Psychological Research, 39, 266-275.

Pascalis, O., \& Bachevalier, J. (1998). Face recognition in primates: a cross-species study. Behavioural Processes, 43, 87-96.

Paukner, A., Anderson, J.R., \& Fujita, K. (2004) Reaction of capuchin monkeys (Cebus apella) to multiple mirrors. Behavioural Processes, 66, 1-6.

Sagiv, N., \& Bentin, S. (2001). Structural encoding of human and schematic faces: Holistic and part based processes. Journal of Cognitive Neuroscience, 13, 937-951.

Sidman, M., \& Stoddard, L.T. (1967). The effects of errors on children's performance on a circle-ellipse discrimination. Journal of the Experimental Analysis of Behavior, 10, 261-270. 